\section{Dr. Kang, et al reply}

\section{To the Editor:}

We thank Dr. Rothschild for his interest ${ }^{1}$ in our article ${ }^{2}$ on the incidence of arterial and venous thrombosis in antineutrophil cytoplasmic antibodyassociated vasculitis (AAV). He raises the interesting issue of susceptibility to both arterial and venous thrombotic events, which is also characteristic of antiphospholipid syndrome. The question arises as to the possible role of antiphospholipid antibodies (aPL) in the thrombotic events seen in AAV. We also thought that this possibility should be examined, but unfortunately our data on aPL are limited, because we studied a retrospective cohort in which these tests were not routinely performed. In fact, we tested for anticardiolipin $\operatorname{IgG}$ and $\operatorname{IgM}$ in only 49 of the 210 patients in the study. We found positive results in 3 patients, none of whom were in the group with thrombosis ${ }^{2}$. In the patients who had a thrombosis, we found negative results in 4 out of the 24 who had an arterial thrombosis, and 5 out of 14 who had a venous thrombosis. These findings were not reported in the original paper because of the low proportion of patients tested and because they were tested only at baseline.

It is possible that some patients may have developed aPL during the course of their disease, which could have gone undetected. Even in the absence of aPL, the high incidence of arterial and venous thrombosis in patients with AAV should encourage us to consider the relative benefits and risks of antiplatelet agents or anticoagulants, as discussed by Rothschild ${ }^{1}$.

AMY KANG ${ }^{\circledR}$, FRACP, MBBS (Hons), Clinical Fellow, Imperial College Renal and Transplant Centre, Imperial College Healthcare National Health Service (NHS) Trust; MARILINA ANTONELOU (1), MRCP, BSc, Academic Clinical Fellow, Imperial College Renal and Transplant Centre, Imperial College Healthcare NHS Trust; NIKKI L. WONG, FRACP, MBBS, Clinical Research Fellow, Imperial College Renal and Transplant Centre, Imperial College Healthcare NHS Trust; ANISHA TANNA, MBBS, BSc, Clinical
Research Training Fellow, Renal and Vascular Inflammation Section, Department of Medicine, Imperial College London; NISHKANTHA ARULKUMARAN ${ }^{\circ}$, PhD, MBBS, Renal Specialty Registrar, Academic Clinical Fellow, Imperial College Renal and Transplant Centre, Imperial College Healthcare NHS Trust; FREDERICK W.K. TAM ${ }^{0}$, PhD, MB BChir, Ken and Mary Minton Chair of Renal Medicine, Renal and Vascular Inflammation Section, Department of Medicine, Imperial College London; CHARLES D. PUSEY (-), DSc, MB BChir, Professor of Medicine, Renal and Vascular Inflammation Section, Department of Medicine, Imperial College London, London, UK. This research is supported by the UK National Institute for Health Research (NIHR) Biomedical Research Centre based at Imperial College Healthcare NHS Trust and Imperial College London. FWT was supported by the Diamond Fund from Imperial Health Charity, and the Ken and Mary Minton Chair of Renal Medicine. MA was supported by the NIHR, and AT by the Wellcome Trust. Address correspondence Dr. C.D. Pusey, Renal and Vascular Inflammation Section, Department of Medicine, Imperial College London, Hammersmith Campus, Du Cane Road, London W12 0NN, UK.

E-mail: c.pusey@imperial.ac.uk

\section{REFERENCES}

1. Rothschild BM. Are thrombotic events in antineutrophil cytoplasmic antibody-associated vasculitis related to the effect of antiphospholipid antibodies? J Rheumatol 2019;46:xxxxx.

2. Kang A, Antonelou M, Wong NL, Tanna A, Arulkumaran N, Tam FW, et al. High incidence of arterial and venous thrombosis in antineutrophilic cytoplasmic antibody-associated vasculitis. J Rheumatol 2019:46;285-93.

First Release June 1 2019; J Rheumatol 2019;46:7; doi:10.3899/jrheum. 190500 